\title{
Reducing power consumption in LEO satellite network
}

\author{
Mohammed Hussein, Abdellatif Abu-Issa, Iyad Tumar, Abdalkarim Awad \\ Department of Electrical and Computer Engineering, Birzeit University, Palestine
}

\begin{tabular}{l} 
Article Info \\
\hline Article history: \\
Received July 5, 2020 \\
Revised Oct 10, 2020 \\
Accepted Dec 5, 2020 \\
\hline
\end{tabular}

\section{Keywords:}

Extended lifetime

LEO satellite

Sleep mode

Traffic matrix

\begin{abstract}
Current low earth orbit (LEO) satellite network display poor power efficiency, running network devices at full capacity all the time regardless of the traffic matrix and the distribution of the population over the Globe. Most of the research on energy efficiency of LEO satellites has focused on component level or link level. Therefore, this kind of research is not holistic to try to look at the satellite system as a single node. To enhance the energy efficiency. The solution should exploits multipath routing and load balancing. LEO network is overprovisioned, and hence selectively shutting down some satellite nodes and links during off-peaks hours seems like a good way to reduce energy consumption. In this paper, we exploit the fact that due to geographical and climatic conditions, some satellite links are expected to be loaded with data while others remain unused. Our approach is to power down satellite nodes and links during period of low traffic, while guaranteeing the connectivity and QoS. Finding the optimal solution is NPproblem and therefore, we explore in this work two heuristic algorithms. We evaluate our heuristics on a realistic LEO topology and real traffic matrices. Simulation results show that the power saving can be significant.
\end{abstract}

This is an open access article under the CC BY-SA license.

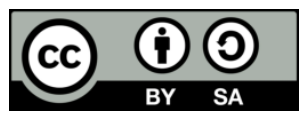

Corresponding Author:

Mohammed Hussein

Department of Electrical and Computer Engineering

Birzeit University

Birzeit, Ramallah, Palestine

Email: mhussein@birzeit.edu

\section{INTRODUCTION}

A low earth orbit satellite (LEO) system generally refers to a large-scale satellite system composed of multiple satellites capable of real-time information processing, wherein the distribution of satellites is called a satellite constellation. LEO satellites mainly used for military target detection, where these satellites are natural to obtain high-resolution images of targets, LEO satellites are also used for mobile phone communication. The low orbital altitude of the satellite makes the transmission delay short and the path loss small. A communication system composed of multiple satellites can achieve exact global coverage. Cellular communication, multiple access, spot beam, and frequency reuse is more efficient other technologies also provide technical support for LEO satellite mobile communications. LEO satellites are the latest and most promising satellite mobile communications systems [1-3]. The LEO satellite constellation consists of multiple satellites in multiple orbits. Because LEO satellites and earth are not synchronized, the constellations are continually changing, and the relative positions of the satellites are always changing. To make real-time communication process easy, satellites must be connected not only to ground terminals and gateway stations but also to satellites themselves. Of course, such links can be connected via terrestrial links or through inter-satellite links (ISLs). The general constellation has multiple satellite orbits for coordination and real-time communication between satellites; there are inter-orbital links between satellites in different orbits [4]. 
These space satellites rely on solar panels and rechargeable batteries as both an energy source and energy storage; these are two sources of energy that complement one another during two uncertain stages. The first stage when the satellite revolves around its orbit and is facing the sun, the solar panels convert the sunlight it absorbs into electrical energy to carry out the vital operations of the satellite, While the remaining power, the system stores it in the batteries for later use. In the second stage, when the satellite is in shadow, it uses the stored energy in the cells to operate the satellite equipment. For example, satellite constellation such as Iridium, satellite, spends about $30 \%$ of its rotation around the orbit in the shadow region [5]. It is impractical or sometimes it is impossible to replace the batteries and hence it is a crucial issue to increase the lifespan of the batteries which eventually will lead to increase the lifetime of the satellite system. Depth of discharge is on of the key factors that determine of a battery. For nickel-hydrogen batteries, as these types of batteries operating in current satellites of the Iridium constellation. Several studies have shown that when the depth of discharge (DoD) is reduced by $15 \%$, this doubles the battery life [6, 7]. Also, lithium-ion batteries are similar in attitude where this type of cells will operate and power Iridium NEXT constellation satellites $[8,9]$. The LEO constellations have a uniform and symmetric nature, include uniformity and consistency, this ensures that there will be minimum paths between two nodes so there will be several options for routing data between two satellites; therefore, the choice of routing of data between satellites constellation will affect the age of these satellites [10].

Our work is an exploration of how overall network energy consumption might be reduced without the affecting the QoS of the whole network. We propose holistic approach to improve the energy saving on the entire satellite network and not in a single node [11], which has been studied in many researches over years. So our approach focus on the entire constellation that finds the optimal set of network components that must use to satisfy the actual demand. The approach will be presented on section 4 . Centralized approaches that solve ILP optimization problems is complex and inefficient. In fact such approaches must solve NP-hard problem. Heuristic based approaches are introduced in this work. To the best of our knowledge, this is the first work that proposes a holistic approach to enhance the energy saving of the LEO satellite system. This paper extends our previous work that has been presented in [12]. Realistic data has been used to explore the performance of the proposed approaches. Both approaches avoid using the satellites located in the dark part of the globe, and therefore give a penalty when using a satellite in that such location. The results illustrates the effectiveness and the good performance the proposed approach. For instance, fast greedy heuristic $(\mathrm{FGH})$ and geographic greedy heuristic (GGH) heuristics can increase the percentage of power saving in the constellation $23 \%$ and $51 \%$, respectively. This way, there would be less use of the battery and decrease in the deception of the discharge and therefore increase the lifespan of the battery and increase the lifetime of the satellite system. The cost of this energy saving is a small delay in the routing between source and destination. The remaining of this paper is organized as follows. Related works is presented in section 2. The architecture of LEO constellations are presented in section 3. Traffic engineering is presented in section 4. In section 5, we formulate the NP-complete optimization problem and in section 6, we describe several heuristics that solve it. Section 7, details the results obtained. Finally, conclusion are drawn in section 8 .

\section{RELATED WORK}

There is a large body of work on power management in contexts complementary to ours. This includes operating system techniques to extend lifetimes in mobiles, and maximizing battery life routing in wireless ad hoc networks. In LEO satellite constellations there are a lot of work in how to routing the data from one satellite to another. The authors in [11] proposed energy control approach in single satellite not in the whole constellation. In addition, the authors in [13] proposed a new optimization approach to reduce the power consumption in single satellite; this approach depend on Lyapunov optimization.

Data transmission is the most power-hungry component of the satellite system. Therefore, it is important to reduce the data transmission from the eclipsed nodes. Smart data transmission from these nodes can enhance the depth of discharge and hence can significantly increase the lifespan of the batteries. QoS issues such as latency and data rates should not affected. Unlike MANET and WSN, the location of nodes of satellite systems is deterministic and not random. This information has been exploited in our previous work [12] and two new routing metrics were presented LASER and SLIM. The basic mechanism employed by both metrics is to disfavour routing data over satellites that have spent the most time in the earth's umbra, in order to reduce using the batteries, without stretching the paths too much to limit the penalty in performance. The location of any satellite can be computed exactly and so can if a satellite is eclipsed, and if yes, for how long time spent in the eclipse. In [14], network connectivity has been utilized to shut down some links. Yet the work in [14] does not take into consideration the traffic distribution among satellites. A Q-learning algorithm is proposed to the power allocation problem in LEO satellite-to-ground communication. In [15] the 
authors proposed a spatial group depending on the optimal uplink power control scheme to increase the performance of random access (RA) in satellite networks.

Many papers addressed the problem reducing the energy consumption in the whole networks. Unfortunately, these papers ignore how to reduce the overall energy consumption in LEO satellite. In [16] the authors discussed the benefits of turning off the network elements (nodes, links) and its impact on the network protocols. They evaluated the total energy consumption for entire network by considering the network topology. To measure the energy consumption for entire network, the authors introduced two scenarios as follows: i) turn off all network devices, ii) turn on only the minimum number of devices that make the network services are available. The power consumption of each scenario was evaluated using simple network topologies. However, the authors showed that increasing the number of network devices may increase the solutions complexity, therefore the problem becomes very expensive to solve even for simple infrastructure. In [17], the authors proposed several heuristics approaches in reducing power consumption in IP wired networks. In [18] closed-form expression for the optimum radio capacity of a spread spectrum noncellular CR system, when operating in a Rayleigh fading condition has been derived.

As we mentioned, there is a common denominator among all the above routing protocols, which is attention only to performance and the speed of data delivery from its source to its final destination without looking at the energy file, so that the general understanding was that these satellites depend on solar energy for operation and there are batteries that support vital operations, and that these batteries can be recharged, so the interest in consuming energy when building these protocols was not worthwhile. However, the constellation Iridium type satellite can be in the shadow area $30 \%$ of the time, making the batteries necessary to operate it. As these batteries are recharged, the depth of discharge will greatly affect their life. The distribution of population over the globe is not constant. For instance, it is almost zero overseas and oceans (around $70 \%$ of the earth), very high inside cities, and middle in the suburbs and rural areas [19]. Therefore, there is a need for an energy aware protocol which takes the whole constellation not a single device. Therefore, an integer linear programming (ILP) model for LEO satellite networks and a two heuristics are presented to minimize the energy consumption of eclipsed satellite nodes. This can reduce the depth of discharge and eventually increase the lifespan of the batteries onboard the satellites.

\section{SATELLITE NETWORK MODEL}

In this section, we present the satellite topology, then we take advantage of the deterministic movement of the satellites in such topology to determine the shadow conditions for each satellite. We use this information in the next sections when designing the new pruning schemes. Figure 1 depicts the LEO satellite architecture. The size of a LEO constellation is NL×ML, where NL is the number of the orbits in the constellation and ML is the number of satellites per orbit [20]. Each satellite has four inter-satellite links (ISLs): two intra-plane ISLs and two inter-plane ISLs. Let $\mathrm{G}=(\mathrm{N}, \mathrm{L})$ represents a graph for a particular satellite network where $\mathrm{N}$ is the satellite nodes (vertices) of the network, and $\mathrm{L}$ is the links (edges) that connect the nodes together in the satellite network. Indeed, the nodes of the satellite network can be divided into two categories based on their spatial location by checking whether the node locates in sun region or eclipse region.

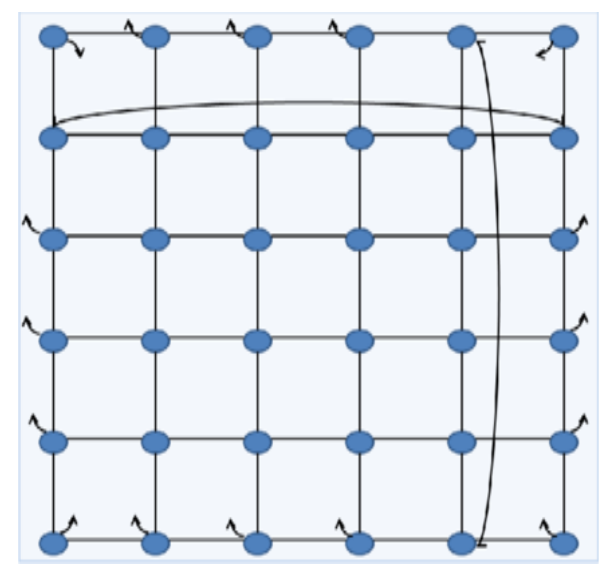

Figure 1. Satellite network topology. Each satellite has four inter-satellite links (ISLs): Two intra-plane ISLs and two inter-plane ISLs 


\section{TRAFFIC MODELING}

The traffic engineering is widely studied over wired networks, however, there are few researches studied the traffic modelling over LEO satellite. Although, the traffic engineering over LEO satellite is more important to increase the availability of such costly constellations. In [21], the authors presented a traffic modelling over LEO constellation. As explained in Figure 2 the globe is divided into $6 \times 12$ cells; each cell occupies $30^{\circ}$ latitude and $30^{\circ}$ longitude. The demand of the traffic in each cell is proportional to the time of this cell in one year. Because the IP traffic is asymmetry, the behaviour of the user and the host are different for each zone. Two databases for user density level $u_{i}$ (million minutes/year) and host density level $h_{i}$ for each zone are created. The user density produces the amount of source requests in every separated zone and the host density represents the host distribution over the geographic zones.

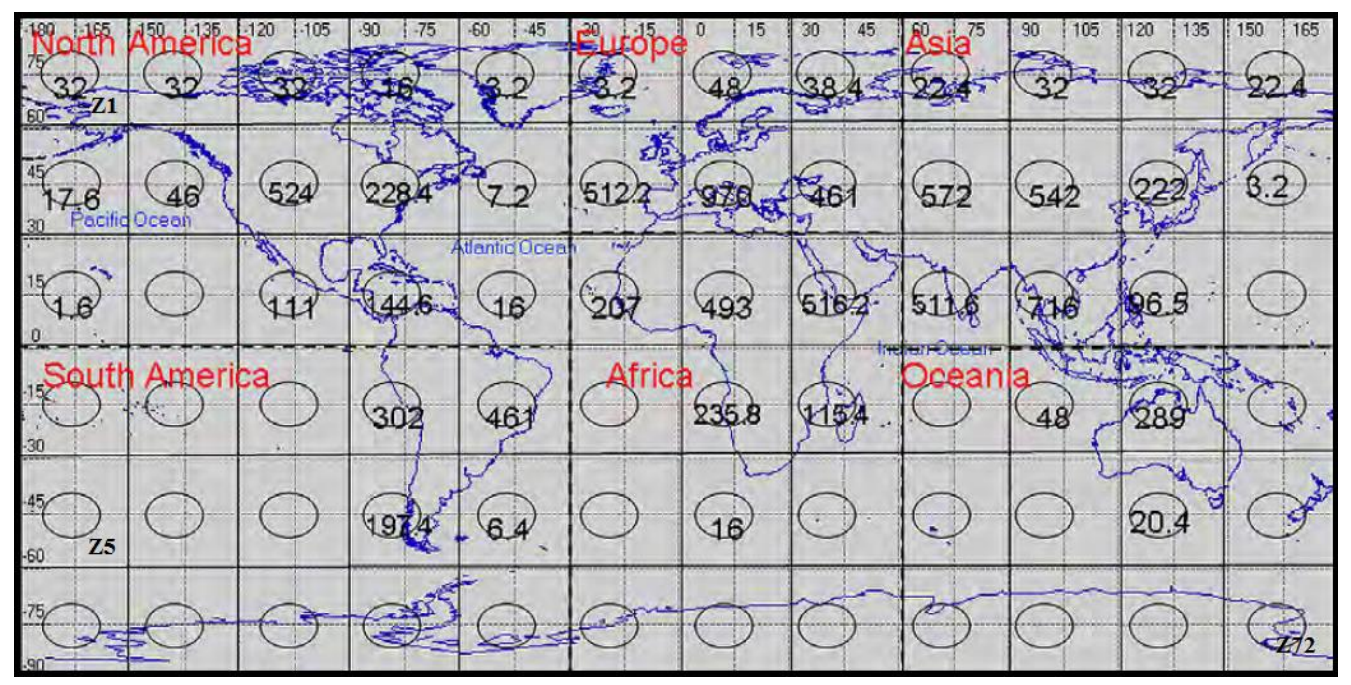

Figure 2. Earth zone division and user density levels $u_{i}$ (million minutes/year)

The basic unit for the time is hour during their research. Each user has different activities during the day, therefore to make the traffic prediction more accurate, the traffic engineering for 24 hours over LEO satellite is shown in Figure 3. The authors assume that the daily evolution of the traffic per user is fit for all user in the world, for each zone the local time is equal to the solar time of the respective zone's center longitude. The annual traffic for year 2005 provides in Figure 3. Assuming that the traffic matrix distribution is the same everyday of the year, so the traffic of each cell for every day is equal to annual traffic/365.

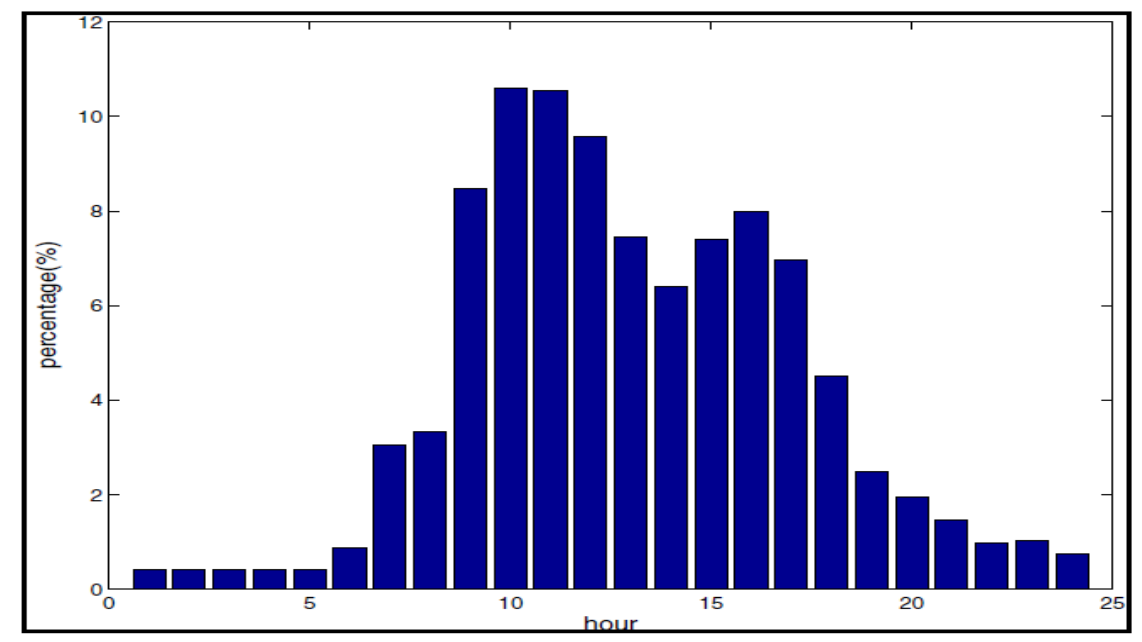

Figure 3. The laws of the traffic demands of LEO satellite network users in $24 \mathrm{~h}$ 
According to these data, the host level $h_{j}$ of zone $Z_{j}$ can be obtained by (1) [22]:

$$
h_{j}=\frac{u_{j}}{\sum_{i} u(i)} \cdot N_{h}(K)
$$

where, $\sum_{i} u(i)$ is the sum of users' density levels for all zones in continent k. $N_{h}(K)$ is the number of hosts in the continent $\mathrm{k}$. Assume that continent k's percentage share of host density on the globe is equal to $\mathrm{p}(\mathrm{k})$ as shown in Table 1.

Table 1. Internet hosts distribution by continent [22]

\begin{tabular}{ccc}
\hline Continent $\mathrm{k}$ & $\mathrm{N}_{\mathrm{h}}(\mathrm{k}) 10^{3}$ & $\mathrm{p}(\mathrm{k}):(\%)$ \\
\hline North America & 71871.5 & 71.27 \\
Europe & 17698 & 17.55 \\
Asia & 7686.4 & 7.62 \\
Oceania & 1873.65 & 1.86 \\
South America & 1474.8 & 1.46 \\
Africa & 241.9 & 0.24 \\
\hline
\end{tabular}

The traffic condition $\left(h_{d}\right)$ from zone $Z_{i}$ to zone $Z_{m}$ is proportional with the users density $u_{i}$, host density $h_{m}$, and the distances between centers of the two zones $d\left(Z_{i}, Z_{m}\right)$.

$$
h_{d}\left(Z_{i}, Z_{m}\right)=\frac{\left(u_{i} \cdot h_{m}\right)^{\alpha}}{\left(d\left(Z_{i}, Z_{m}\right)\right)^{\beta}}
$$

Distance between two zones can be calculated using the longitude and latitude for the center of that zones. " $\alpha=0.5 \beta=1.5$ [22]. To calculate the generated traffic engineering to practical wideband LEO satellite network, we use $h_{d}$ as the coefficient for obtaining the average traffic values $H_{d}$ between two satellite nodes".

$$
H_{d}=\frac{h_{d}\left(Z_{i}, Z_{m}\right)}{\sum_{\forall Z_{i}} \sum_{\forall Z_{m}} h_{d}\left(Z_{i}, Z_{m}\right)} X \frac{\text { total offered traffic }}{3600} X \frac{\alpha_{h}}{100}
$$

Total offered traffic is the total amount of traffic generated worldwide per day, $\mathrm{h}$ is the current local time, Figure 3 shows $a_{h}$ is the activity percentage in the corresponding hour. The traffic demand depends on the location as well as the time.

\section{PROBLEM FORMULATION}

We have employed graph theory to represent the problem. The network is represented as a set of nodes or vertices $(\mathrm{V})$ and edges $(\mathrm{E})$. The node represents a satellite and an edge represents a link between two satellites, i.e. $\mathrm{G}=(\mathrm{V}, \mathrm{E})$. The set $\mathrm{N}=|\mathrm{V}|$ represent the set of satellites and the links set $\mathrm{L}=|\mathrm{E}|$ represents the links set. The link capacity cuv represents the capacity of a link between the two nodes u, and v, i.e., the link $(\mathrm{u}, \mathrm{v}) \in \mathrm{E}$ an the nodes $\mathrm{u}, \mathrm{v} \in \mathrm{V}$. The variable $\alpha \in\{0,1\}$ represents the maximum link utilization. (MLU) that can be tolerated when routing data between vertices suppose $H_{d}^{s d}$ be the average amount of traffic that goes from satellite node $s=1, \ldots, N$ to satellite node $d=1, \ldots, N$. Let $D$ be the collection of all these demands in the topology. Let $\mathrm{x}_{\mathrm{uv}}$ be a binary variable to decide if the link is used or not, when a satellite link $(\mathrm{u}, \mathrm{v})$ is used $\left(\mathrm{x}_{\mathrm{uv}}=1\right)$. Also, let $\mathrm{y}_{\mathrm{u}}$ be binary variables take the value of 1 if the satellite node $\mathrm{i}$ is work. Let $f_{u v}^{s d}$ be the amount of flow from source $(s)$ to destination $(d)$ that is routed through the arc from $u$ to v. Similarly, let $f_{u v}$ be the total amount of traffic routing on the link from $u$ to $v$. The power consumption of satellite node is $p_{u}$ and satellite link is $\mathrm{p}_{\mathrm{uv}}$ The ILP optimization problem can be formulated as seen below.

Minimize

$$
P_{t o t}=\sum_{(u, v) \in E} p_{u v} x_{u v}+\sum_{u \in V} p_{u} y_{u}
$$

Subject to

$$
\sum_{v=1}^{N} f_{v u}^{s d}-\sum_{v=1}^{N} f_{u v}^{s d}=\left\{\begin{array}{cc}
H_{d}^{s d} & \forall s, d, u=s \\
-H_{d}^{s d} & \forall s, d, u=d \\
0 & \forall s, d, u \neq s, d
\end{array}\right.
$$




$$
\begin{aligned}
& \sum_{d \in D}\left(f_{u v}^{d}+f_{v u}^{d}\right) \leq \alpha c_{u v} x_{u v} \quad \forall u, v \\
& \sum_{v=1}^{N} x_{u v}+\sum_{v=1}^{N} x_{v u} \leq M y_{u} \quad \forall u
\end{aligned}
$$

As shown in (4) specifies the consumed power in active satellite nodes and links. The constraint in (5) explain the standard conservation flows, flows that leave the source satellite or entering a destination satellite node of demand $\mathrm{d}$ sum to be $\mathrm{H}_{\mathrm{d}}$. In (6) the load in every link must be smaller than link capacity. In (7) explain when the satellite node can be turned off, if all incoming and outgoing ISLs are turned off. Finally, $\mathrm{M}$ in (7) is a constant value for maximum number of links connected to a node.

The formalization mentioned in (4) to (7) is an NP-hard problem, which is classified in "capacitated multi-commodity minimum cost flow problems (CMCF)" [23]. Such problems can have exact solution for trivial cases. For this reason we propose two heuristics to solve this optimization problem. Moreover, the energy saving achieved by switching off any satellite node is higher than switching off single inter-satellite link. Algorithms therefore try to turn off satellite nodes first, and then the same algorithms are used to turn off ISLs.

\section{EFFICIENT HEURISTICS}

The sleep-scheduling approach selects a set of satellite nodes and links to be switched off during the off-peak hours, to save more energy in the constellation network. The main objective of sleep scheduling is to find the minimum connected sub network that consumes the least amount of energy while satisfying the performance matrices of the network. The optimal solution for the specific network is NP-hard problem and therefore a time consuming computational problem. Therefore, we propose two efficient heuristics algorithms to solve the problem in an acceptable time. The general idea of these heuristics are removing network nodes and links in certain order until no elements can be removed. First, the two heuristics start by considering all the network elements are powered on, hence xuv $=1 \forall$ uv for every actual link in LEO topology, and yu $=1 \forall \mathrm{u}$. Then, the algorithm check iteratively if a given element can be removed or not.

\subsection{Fast greedy heuristic (FGH)}

The first heuristic approach proposed in this paper is called fast greedy heuristic (FGH). This approach removes the maximum number of satellite nodes and links without taking in its consideration the location of the satellite in eclipse or sun. FGH algorithm in each iteration removes nodes and links from the network graph then traffic is rerouted in the frugal graph. After rerouting the traffic if violation is occurred then the specific node or link is powered on again. Figure 4 shows the algorithm of the operation of FGH.

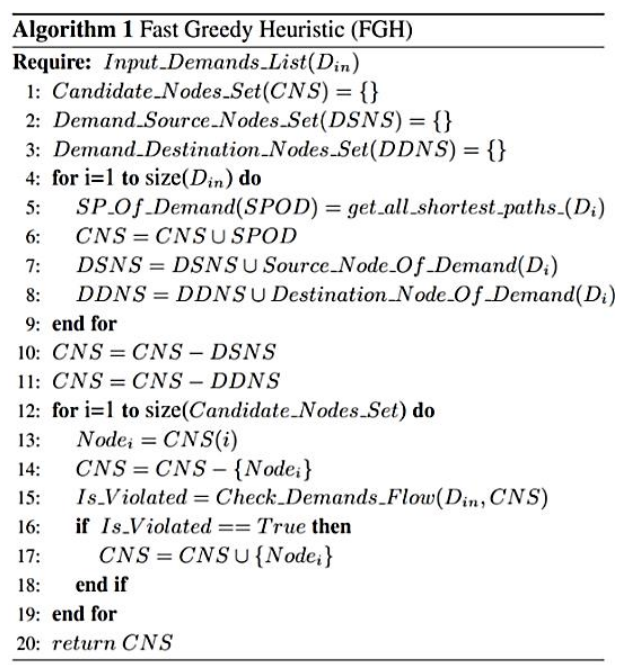

Figure 4. Algorithm of the operation of FGH

Note: Similar procedure (FGH) is used to switch off satellite links. So, we do not need to repeat writing the algorithm. The solution of FGH applied to switch off satellite nodes and links that provide the overall power saving in LEO topology. 


\subsection{Geographic greedy heuristic (GGH)}

Unlike other networks such as mobile networks, ad hoc networks and sensor networks where battery lifetime is also vital, LEO satellite moves in a deterministic path. Each satellite in such constellation can be computed if it is in sun or eclipse and how much time spent in eclipse [24]. This characteristic has been not exploited in FGH algorithm. Therefore, the next heuristic improves on FGH is by making more careful choices by preferring shutting satellite nodes and links exposed to eclipse than to sun.

GGH heuristic improves FGH by switching off satellite nodes that located in eclipse rather than in sun. These space satellites rely on solar panels and rechargeable batteries as both an energy source and energy storage; these are two sources of energy that complement one another during two uncertain stages. The first stage when the satellite revolves around its orbit and is facing the sun, the solar panels convert the sunlight it absorbs into electrical energy to carry out the vital operations of the satellite, while the remaining power, the system stores it in the batteries for later use. In the second stage, when the satellite is in shadow, it uses the stored energy in the cells to operate the satellite equipment. For example, satellite constellation such as Iridium, satellite, spends about $30 \%$ of its rotation around the orbit in the shadow region. It is difficult and sometimes impossible to replace the batteries of the satellite. Increasing the lifetime of the batteries inevitably leads to an increase in the lifetime of the satellite itself. Depth of battery discharge (DoD) is one of the key factors that determine the lifespan of a battery. For instance, the life span of nickel-hydrogen batteries which are used in current satellites of the Iridium constellation can be improved significantly when reducing the DoD. A $15 \%$ reduction of DoD is can double the battery life $[8,9]$. GGH is designed to put in sleep mode satellite nodes and links exposed to eclipse rather than to sun, then reducing the power consumption in satellite batteries. Therefore, GGH heuristic tries only to shut down as much as possible satellites nodes and links that located in earth's umbra.

Most of population is live at the Northern Hemisphere, especially between $0^{\circ}$ and $50^{\circ} \mathrm{N}$. Moreover, traffic generated on the Southern Hemisphere is very light compared with the Northern Hemisphere [25]. Encouraged by this fact, GGH heuristic first try to switch off eclipse satellite nodes, links that located in Southern Hemisphere, then try to switch off the eclipse nodes, and links in the Northern Hemisphere. Since, it is preferred to remove firstly nodes and links that are not involved in many shortest paths than over utilized nodes. The metacode for GGH is presented in algorithm 2 in Figure 5.

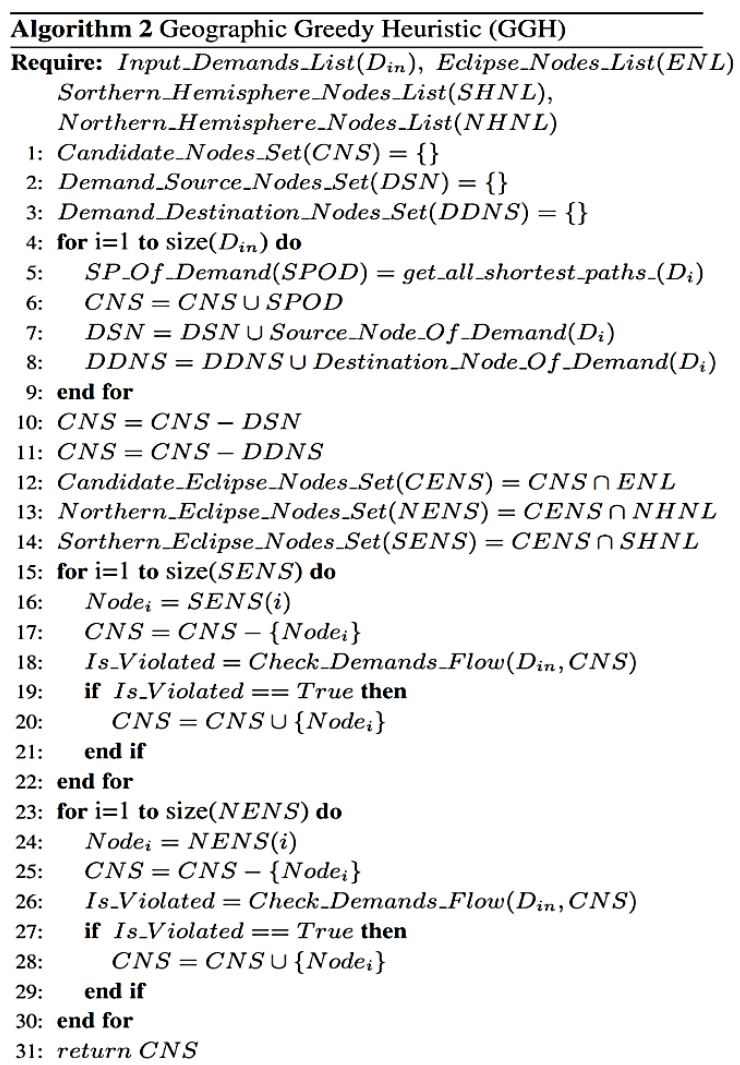

Figure 5. The metacode for GGH 
Note: Similar procedure $(\mathrm{GGH})$ is used to switch off some satellite links. So, we do not need to repeat writing the algorithm for shutting down the links. The solution of GGH applied to switch off satellite nodes and links provide the overall power saving in LEO satellite constellation topology.

\section{EXPERIMENTAL EVALUATION}

In this section, we evaluate the performance of FGH and GGH in terms of power saving and end to end delay using real topologies and their traffic matrices.

\subsection{Experimental setup}

In this paper the ILP formulation is solved using CPLEX version 12.6. While the two heuristics are implemented using custom simulator in MATLAB. The LEO satellite constellation explained in section 3 is used during the simulation. The goal is to put some nodes in sleep mode and keep the network fully connected. We also keep a low link utilization to indicate that the QoS is untouched. We tested the performance of FGH and GGH algorithms during peaks and off-peaks hours. The satellite link capacity is $155 \mathrm{Mbps}$. The LEO satellite link utilization $\alpha$ is set to 0.5 . The "total offered traffic" is set to be $500 \mathrm{~Tb} / \mathrm{day}$ which represents a real traffic discussed in section 4 . Also, the local time $(\mathrm{h}) \in[8 \mathrm{am}, 6 \mathrm{pm}]$. We have assumed that the satellites located on the sunny part does not use the battery and hence their power consumption is set to zero. Whereas, we have assumed that the satellites located in the dark part consumes 1000 Watt. The link consumption is set to 50 Watt for links in the dart part and 0 for the links in the sunny part.

A multicore Linux machine has been used to run the optimization problem. The machine has 16 Giga byte RAM and it has $83.4 \mathrm{GHz}$ processor. To find the optimal solution using CPLEX takes several hours even for the smallest network (e.g., Atlanta network, 15 nodes and 22 links), therefore, we force CPLEX to stop after 3 hours of execution. Considering the fact that, limit the computation time to 300 seconds only in CPLEX can obtain around $96 \%$ of the optimal power saving. The time complexity in average for FGH and GGH heuristics to identify a satellite node for removal after examining a constant number of nodes, resulting in $\mathrm{O}(|\mathrm{N}|)$ executions of the linear program.

\subsection{Energy saving}

First, we run 10 traffic matrices using equation explained in section 4, to measure first the power saving obtained from the optimal solution and from FGH and GGH. Power saving is calculated using the following:

$$
\text { saving }=100\left(1-\frac{\sum_{(u, v) \in E} p_{u v} x_{u v}+\sum_{u \in V} p_{u} y_{u}}{P_{\text {tot }}}\right.
$$

Figure 6 illustrates the power saving obtained from LEO satellite topology with ten different traffic matrices. CPLEX is able to obtain the maximum power saving however, GGH is outperform FGH, since it takes the location of the satellite in its consideration. As power consumption decreases in eclipsed satellite batteries, the DoD will be decreased also in the satellites batteries. This will lead to increase the service life time of LEO satellite constellations. Moreover, more energy efficient network architectures would allow LEO network deployments in less developed countries.

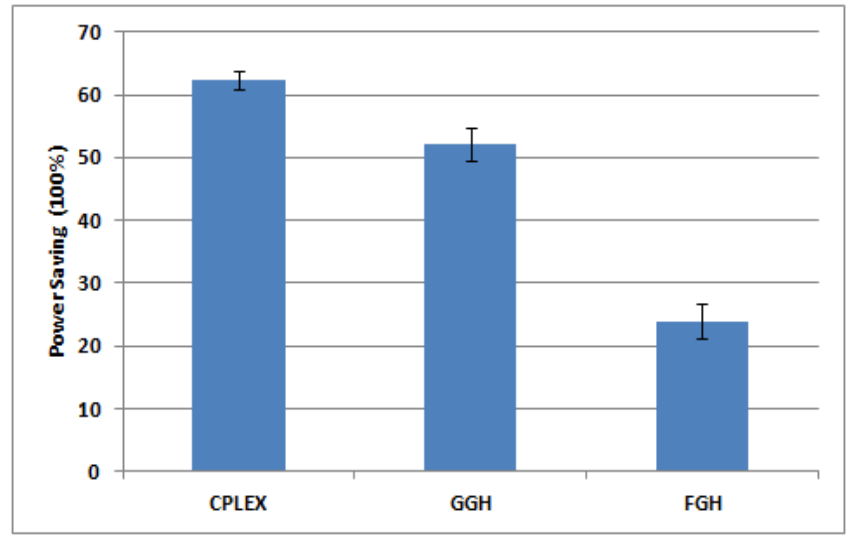

Figure 6. Percentage of power saving using 10 traffic matrices 


\subsection{Daily traffic variation}

In Figure 7 we evaluate the possible power saving in a scenario in which the traffic is changed during night and day. In particular, more power saving is possible when the network is lightly loaded, i.e., during night. Figure 7 display the percentage of power saving for FGH and GGH heuristics. During the night since the traffic is so little the achieved power saving is the absolute maximum. During GGH heuristic outperforms FGH since GGH takes into its consideration the location of the satellites in eclipse or sun. However, FGH heuristic turn off the maximum number of satellite nodes without taking the location of the satellite in its consideration.

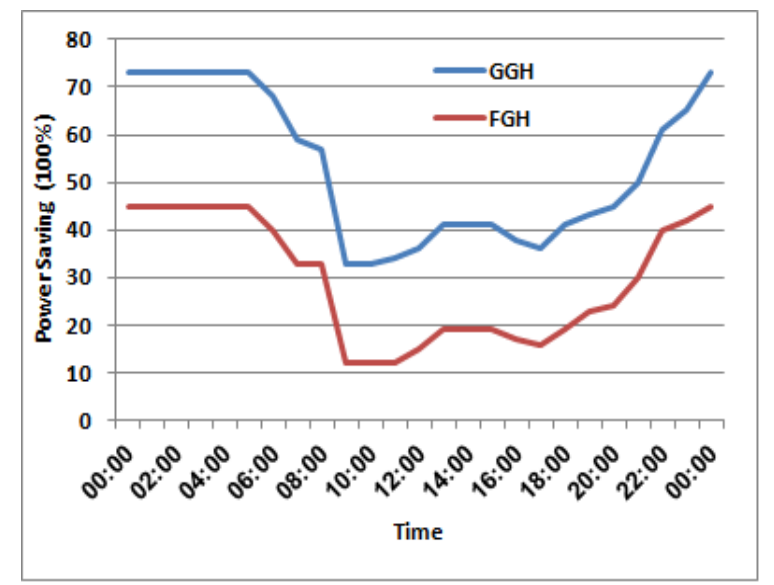

Figure 7. Power saving on the LEO satellite topology in $24 \mathrm{~h}$. During the day, the power saving decreases as the traffic increases since more capacity is required to guarantee the QoS constraint. Saving is practically constant during the night since the traffic is so little

\subsection{Stretch factor}

Stretch factor is defined as the ratio between the average route length (in hops) in the new topology divided by the average route length with the original topology (using all network nodes and links). To test the reaction of stretch factor we generated 10 traffic matrices using the traffic model in section 4 . We first route all flows in the original topology using Dijkstra shortest path, then we use the same flows to calculate the average path length in the new topology (after removing a set of links). Results for the LEO topology with the 10 traffic matrices are given in Figure 8, we see that the impact of route length is very limited. E.g., the average increase is $1.19 \%$ for GGH heuristic. Figure 8 shows that GGH leads to a much better stretch factor when compared to FGH. This is to be expected since GGH heuristic turn off less number of satellite links and nodes than FGH. However, GGH heuristic significantly outperforms the FGH heuristic with regards to power savings also. Because, GGH turn off more eclipsed satellite nodes compare to FGH algorithm.

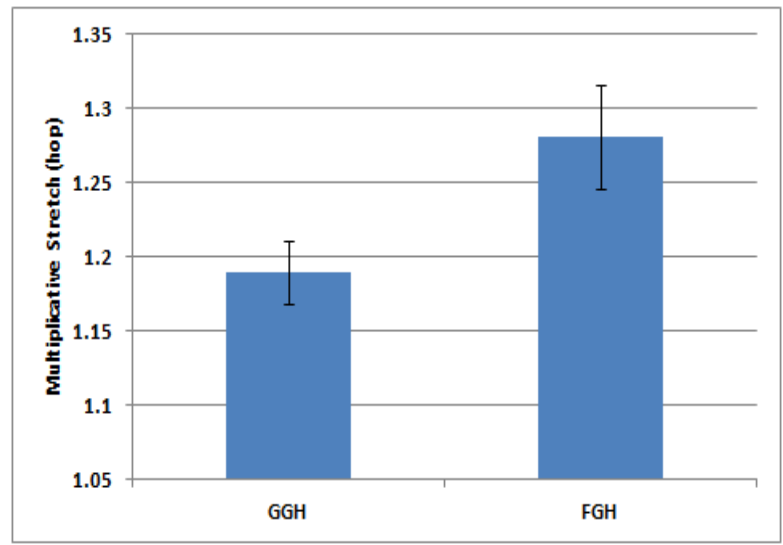

Figure 8. Multiplicative stretch in hops for the proposed heuristics (average multiplicative stretch factor) 


\section{CONCLUSION}

At the end of this paper, we conducted a comprehensive study on satellites, especially those in lowEarth orbit. These satellites are the new generation of communications due to their distinctive characteristics. In this paper, we formulated the problem of energy consumption as ILP formulations, showing that the problem falls in the class of NP-problem. Then we adopted two heuristics algorithms to solve the problem. The two heuristics, FGH and GGH are able to reduce the energy consumption in any LEO constellation by a considerable value. The effect of energy is significant and not negligible, especially with LEO satellites, this is due to the difficulty of maintaining it or changing its battery. As for recommendations for the future, we have to make more comprehensive studies and put other factors to increase the life of the satellites, taking into account the increase in the battery life and also reduce the error rate in the packet delivery ratio in order to increase the speed of data exchange in addition to maintain the age of the satellite.

\section{REFERENCES}

[1] T. Taleb, et al., "Explicit load balancing technique for NGEO satellite IP networks with on-board processing capabilities," IEEE/ACM Transactions on Networking, vol. 17, no. 1, pp. 281-293, 2009.

[2] H., Mohammed, et al., "Location-aware load balancing routing protocol for LEO satellite networks," 2018 Int. Conf. on Advanced Communication Technologies and Networking (CommNet), Marrakech, 2018, pp. 1-7.

[3] D. Sakshi, and D. Kumar, "A review of remotely sensed satellite image classification," International Journal of Electrical and Computer Engineering, vol. 9, no. 3, pp. 1720-1731, 2019.

[4] A. Ferreira, J. Galtier, and P. Penna, "Topological design, routing and handover in satellite networks," Handbook of wireless networks and mobile computing, pp. 473-493, 2002.

[5] S. Q. Kidder and T. H. V. Haar, "A satellite constellation to observe the spectral radiance shell of earth," Proceedings of 13th Conference on Satellite Meteorology and Oceanography, 2004.

[6] L. H. Thaller and T. P. Barrera, "Modeling performance degradation in nickel hydrogen cells," DTIC Document, Tech. Rep., 1991.

[7] A. Dutta and Y. Yemini, "Power management of LEOs under bursty broadband traffic," 17th AIAA International Communications Satellite Systems Conference and Exhibit, Feb. 1998, pp. 1-11.

[8] J. -W. Lee, Y. K. Anguchamy, and B. N. Popov, "Simulation of charge discharge cycling of lithium-ion batteries under low-earth-orbit conditions," Journal of Power Sources, vol. 162, pp. 1395-1400, 2006.

[9] G. Ning and B. N. Popov, "Cycle life modeling of lithium-ion batteries," Journal of The Electrochemical Society, vol. 151, no. 10, pp. A1584-A1591, 2004.

[10] "Iridium next: A global effort to launch the future of global communications," Iridium Press release, Aug. 2013

[11] A. C. Fu, E. Modiano, and J. N. Tsitsiklis, "optimal energy allocation and admission control for communications satellites," IEEE/ACM Transactions on Networking, vol. 11, no. 3, pp. 488-500, 2003.

[12] M. Hussein, G. Jakllari, and B. Paillassa, "On Routing for Extending Satellite Service Life in LEO Satellite Networks (regular paper)," IEEE Global Communications Conference (GLOBECOM), 2014, pp. 2832-2837.

[13] W. Fang, et al., "Energy-efficient network transmission between satellite swarms and earth stations based on lyapunov optimization techniques," Mathematical Problems in Engineering, vol. 2014, 2014.

[14] H. Mohammed, I. Elayyan, and W. Ghanem, "Topology-aware approach for reducing power consumption in LEO satellite constellations," International Conference on Advanced Communication Systems and Information Security (ACOSIS), 2016.

[15] Zhao, Bo, et al., "Spatial Group Based Optimal Uplink Power Control for Random Access in Satellite Networks," IEEE Transactions on Vehicular Technology, 2020.

[16] M. Gupta and S. Singh, "Greening of the internet," Proceedings of the 2003 conference on Applications, technologies, architectures, and protocols for computer communications, ACM, 2003, pp. 19-26.

[17] L. Chiaraviglio, M. Mellia, and F. Neri, "Minimizing isp network energy cost: formulation and solutions," IEEE/ACM Transactions on Networking (TON), vol. 20, no. 2, pp. 463-476, 2012.

[18] V., Panagiotis, "Estimation of radio capacity of a spread spectrum cognitive radio Rayleigh fading system," Proceedings of the 17th Panhellenic Conference on Informatics, 2013.

[19] A. Fatih, O. Korcak, and A. Jamalipour, "Exploring the routing strategies in next-generation satellite networks," IEEE Wireless Communications, vol. 14, no. 3, pp. 79-88, 2007.

[20] H. Liu and F. Sun, "Routing for predictable leo/meo multi-layered satellite networks," Foundations and Practical Applications of Cognitive Systems and Information Processing. Springer, 2014, pp. 511-522.

[21] W. Jiang and P. Zong, "A discrete-time traffic and topology adaptive routing algorithm for leo satellite networks," Int'l J. of Communications, Network and System Sciences, vol. 4, no. 1, p. 42, 2011.

[22] C. Chen and E. Ekici, "A routing protocol for hierarchical leo/meo satellite IP networks," Wireless Networks, vol. 11, no. 4, pp. 507-521, 2005.

[23] I. Ghamlouche, T. G. Crainic, and M. Gendreau, "Cycle-based neighbourhoods for fixed-charge capacitated multicommodity network design," Operations research, vol. 51, no. 4, pp. 655-667, 2003.

[24] C. R. O. Longo, S. L. Rickman, and L. B. J. S. Center, "Method for the calculation of spacecraft umbra and penumbra shadow terminator points," National Aeronautics and Space Administration, vol. 3547, 1995.

[25] M. Mohorcic, et al., "Adaptive routing for packet-oriented intersatellite link networks: Performance in various traffic scenarios," IEEE Transactions on Wireless Communications, vol. 1, no. 4, pp. 808-818, 2002. 